\title{
Design of Neural Network-based Backstepping Controller for the Folding-Boom Aerial Platform Vehicle
}

\author{
Hu Haidong \\ school of information engineering \\ Inner Mongolia University of Science and Technology, \\ Baotou, China \\ e-mail: haidong.hu@126.com
}

Ren Yan

school of information engineering

Inner Mongolia University of Science and Technology, Baotou, China

e-mail: renyan.ry@163.com

\begin{abstract}
In this paper, the robust trajectory tracking problem is addressed for the work platform of folding-boom aerial platform vehicle in the presence of uncertainties and disturbances. The control objective is to make the work platform move along a desired reference trajectory and make the vibration inhibit at the same time. Since neural network system can approximate any nonlinear function with arbitrary accuracy over a compact set in the light of the universal approximation theorem, a neural network-based backstepping controller, which composed of backstepping control and neural network, is proposed for the trajectory tracking control of the work platform in the case of modeling uncertainties and disturbances. According to Lyapunov stability theorem, the stability and convergence of the overall system can be guaranteed by the derived control law. In addition, simulation results demonstrate that the proposed controller is effective for suppressing the vibration and reducing trajectory tracking error of the work platform.
\end{abstract}

Keywords-aerial platform vehicle; model uncertainties; trajectory tracking control; backstepping controller; neural network

\section{I、INTRODUCTION}

Folding-boom aerial platform vehicle is a kind of device which can lift people to the height for installation or maintenance [1], the scheme of which is shown in Figure.1.

As it requires vey high safety, both the stability of movement and the accuracy of positioning of work platform should be guaranteed.

Since the light-long beam is widely used in the arm system, the influence of elastic deformations of beam should be considered. Therefore, in the literature [1], the beams are seen as flexible and the dynamics equations of the arm system of folding-boom aerial platform vehicle are set up based on flexible multi-body dynamics theory and Lagrange's equation. The establishment of the model

\author{
Liu Huibo \\ school of information engineering \\ Inner Mongolia University of Science and Technology, \\ Baotou, China \\ e-mail: liuhuibo2002@126.com
}

lays foundation of the research of steady movement and accurate positioning of work platform.

Due to the robust performance, integrator backstepping control has been applied to many nonlinear systems successfully, such as single link flexible manipulator [2] and multiple link rigid manipulator [3], etc. In addition, in [4], the backstepping control scheme has been used for the control of work platform of folding-boom aerial platform vehicle effectively. However, this method can only be used for the accurate dynamic model of the arm system of folding boom aerial platform vehicle.

In fact, there exist various uncertainties due to external disturbances and approximation of the modeling [5, 6]. As a result, a robust adaptive control scheme is developed for a class of uncertain nonlinear systems by the combination of backstepping control method and fuzzy control method in [7]. Moreover, as neural network can be used to approximate any nonlinear function over a compact set with arbitrary accuracy [8], it has attracted a wide spread attention. In $[9,10]$, the combination of backstepping design and neural network has been used in the control of uncertain nonlinear systems.

In this paper, a neural network-based backstepping controller, which combines backstepping control with neural network, is proposed for the trajectory tracking control of work platform with modeling uncertainties. As the control law is derived from the Lyapunov stability conditions, the stability and convergence of the overall system can be ensured. Furthermore, simulation results demonstrate the effectiveness of the proposed controller for suppressing the vibration and reducing the tracking error of work platform in the presence of model uncertainties.

The organization of this paper is as follows. Flexible multi-body dynamic model of arm system of folding-boom aerial platform vehicle is presented in Section II. Section III proposes a neural network-based backstepping controller, which is used for trajectory tracking control of work platform. Finally, simulation 
results are illustrated in Section IV, and some conclusions are drawn in Section V.

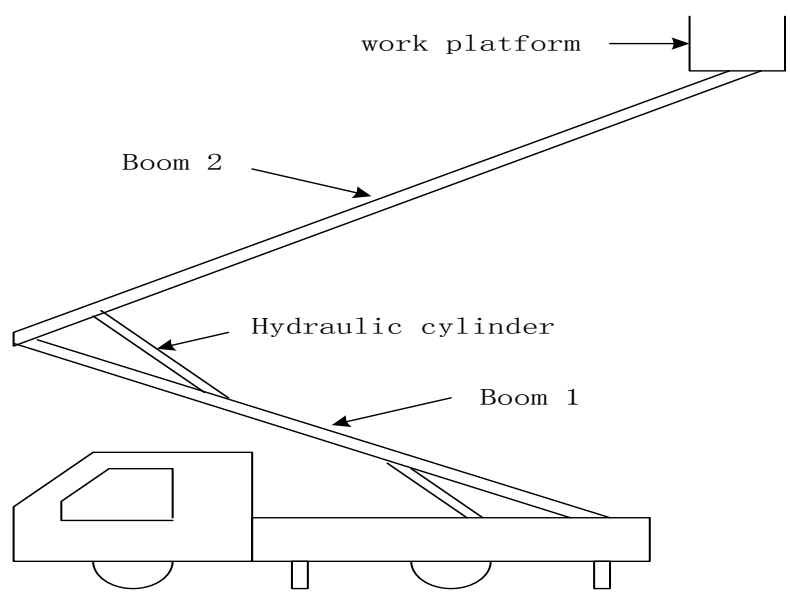

Figure 1. Scheme of folding-boom aerial platform vehicle

\section{II、FleXIBLE Multi-Body DynAmics MODEL OF ARM SYSTEM}

The flexible multi-body dynamics model of arm system is represented by[1]

$$
\left\{\begin{array}{l}
G \ddot{\theta}+U \dot{\theta}^{2}+H \ddot{q}+R=Q_{\theta} \\
M \ddot{q}+N q+H^{T} \ddot{\theta}+V^{T} \dot{\theta}^{2}=Q_{q}
\end{array} .\right.
$$

where, $Q_{\theta}=\left[\begin{array}{ll}Q_{1} & Q_{2}\end{array}\right]^{T}, \quad Q_{q}=\left[\begin{array}{llll}Q_{3} & Q_{4} & Q_{5} & Q_{6}\end{array}\right]^{T}$, $\ddot{\theta}=\left[\begin{array}{ll}\ddot{\theta}_{1} & \ddot{\theta}_{2}\end{array}\right]^{T} \quad, \quad \dot{\theta}^{2}=\left[\begin{array}{ll}\dot{\theta}_{1}^{2} & \dot{\theta}_{2}^{2}\end{array}\right]^{T}$, $q=\left[\begin{array}{llll}q_{11} & q_{12} & q_{21} & q_{22}\end{array}\right]^{T}$, $\ddot{q}=\left[\begin{array}{llll}\ddot{q}_{11} & \ddot{q}_{12} & \ddot{q}_{21} & \ddot{q}_{22}\end{array}\right]^{T}, \quad G, \quad M, \quad H$ are the mass matrix and can be described as follows:

$$
\begin{aligned}
& G=\left[\begin{array}{cc}
\left(m_{1} / 3+m_{2}+m\right) l_{1}^{2} & \left(m_{2} / 2+m\right) l_{1} l_{2} \cos \left(\theta_{1}-\theta_{2}\right) \\
\left(m_{2} / 2+m\right) l_{1} l_{2} \cos \left(\theta_{1}-\theta_{2}\right) & \left(m_{2} / 3+m\right) l_{2}^{2}
\end{array}\right] \\
& M=\operatorname{diag}\left[m_{1} / 2 \quad m_{1} / 2 \quad m_{2} / 2 \quad m_{2} / 2\right] \\
& H=\left[\begin{array}{cccc}
m_{1} l_{1} / \pi & -m_{1} l_{1} / 2 \pi & 2 m_{2} l_{1} / \pi \cos \left(\theta_{1}-\theta_{2}\right) & 0 \\
0 & 0 & m_{2} l_{2} / \pi & -m_{2} l_{2} / 2 \pi
\end{array}\right]
\end{aligned}
$$

$U, V$ and $N$ are the coefficient matrix and can be expressed as

$$
\begin{aligned}
& U=\left[\begin{array}{cc}
0 & \left(m_{2} / 2+m\right) l_{1} l_{2} \sin \left(\theta_{1}-\theta_{2}\right) \\
-\left(m_{2} / 2+m\right) l_{1} l_{2} \sin \left(\theta_{1}-\theta_{2}\right) & 0
\end{array}\right] \\
& V=\left[\begin{array}{cccc}
0 & 0 & -2 m_{2} l_{1} \sin \left(\theta_{1}-\theta_{2}\right) / \pi & 0 \\
0 & 0 & 0 & 0
\end{array}\right] \\
& N=\operatorname{diag}\left[E I_{1} \pi^{4} / 2 l_{1}^{3} \quad 8 E I_{1} \pi^{4} / l_{1}^{3} \quad E I_{2} \pi^{4} / 2 l_{2}^{3} \quad 8 E I_{2} \pi^{4} / l_{2}^{3}\right]
\end{aligned}
$$

$R$ is column vector and is given by

$$
\begin{aligned}
R= & {\left[2 / \pi m_{2} l_{1} \sin \left(\theta_{1}-\theta_{2}\right)\left(\dot{\theta}_{1}+\dot{\theta}_{2}\right) \dot{q}_{21}+\left(m_{1} / 2+m_{2}+m\right) g l_{1} \cos \theta_{1}\right.} \\
& \left.\left(m_{2} / 2+m\right) g l_{2} \cos \theta_{2}\right]^{T}
\end{aligned}
$$

III、THE DESIGN OF NEURAL NETWORK-BASED BACKSTEPPING CONTROLLER FOR FOLDING-BOOM AERIAL PlATFORM VEHICLE

Defining $\quad x_{1}=\left[\begin{array}{lllllll}\theta_{1} & \theta_{2} & q_{11} & q_{12} & q_{21} & q_{22}\end{array}\right]^{T}$, $x_{2}=\left[\begin{array}{llllll}\dot{\theta}_{1} & \dot{\theta}_{2} & \dot{q}_{11} & \dot{q}_{12} & \dot{q}_{21} & \dot{q}_{22}\end{array}\right]^{T}$, then the state equations of (1) can be written as

$$
\left\{\begin{array}{l}
\dot{x}_{1}=x_{2} \\
\dot{x}_{2}=\bar{M}^{-1}\left(Q-\bar{V} x_{2}^{2}-\bar{N} x_{1}-\bar{R}\right)
\end{array} .\right.
$$

where, $\bar{M}=\left[\begin{array}{cc}G & H \\ H^{T} & M\end{array}\right], \quad \bar{M}=\left[\begin{array}{cc}G & H \\ H^{T} & M\end{array}\right], \bar{N}=\left[\begin{array}{cc}0 & 0 \\ 0 & N\end{array}\right]$, $\bar{R}=\left[\begin{array}{ll}R^{T} & 0\end{array}\right]^{T}, \quad x_{2}^{2}=\left[\begin{array}{llllll}\dot{\theta}_{1}^{2} & \dot{\theta}_{2}^{2} & \dot{q}_{11}^{2} & \dot{q}_{12}^{2} & \dot{q}_{21}^{2} & \dot{q}_{22}^{2}\end{array}\right]^{T}$

Choose the reference trajectory as $r(t)=\left[\begin{array}{llllll}r_{1}(t) & r_{2}(t) & r_{3}(t) & r_{4}(t) & r_{5}(t) & r_{6}(t)\end{array}\right]^{T}, \quad$ then $\dot{r}(t)=\left[\begin{array}{llllll}\dot{r}_{1}(t) & \dot{r}_{2}(t) & \dot{r}_{3}(t) & \dot{r}_{4}(t) & \dot{r}_{5}(t) & \dot{r}_{6}(t)\end{array}\right]$ $\ddot{r}(t)=\left[\begin{array}{llllll}\ddot{r}_{1}(t) & \ddot{r}_{2}(t) & \ddot{r}_{3}(t) & \ddot{r}_{4}(t) & \ddot{r}_{5}(t) & \ddot{r}_{6}(t)\end{array}\right]^{T}$.

Defining $z_{1}=x_{1}-r(t)$ as the position tracking error, then the virtual control is given by $\alpha_{1}=-k_{1} z_{1}$, in which, $k_{1}$ is positive constant. Furthermore, defining $z_{2}=x_{2}-\alpha_{1}-\dot{r}(t)$, then the control law $Q$ is designed as

$$
Q=\bar{M}\left[-z_{1}-k_{2} z_{2}-k_{1}\left(x_{2}-\dot{r}(t)\right)+\ddot{r}(t)\right]+\bar{V} x_{2}^{2}+\bar{N} x_{1}+\bar{R} \text {. }
$$

follows: where $k_{2}>0$.

From paper[4], it is concluded that for the dynamics model (2), the control law is designed as (3), then the trajectory tracking error of work platform will converge to zero by choosing suitable design constant $k_{1}$ and $k_{2}$.

In practice, there exist various uncertainties in the model. Considering the model uncertainties, the state equation (2) can be rewritten as follows

$$
\left\{\begin{array}{l}
\dot{x}_{1}=x_{2} \\
\dot{x}_{2}=\bar{M}^{-1} Q+f(x)
\end{array} .\right.
$$

where $f(x)=-\bar{M}^{-1}\left(\bar{V} x_{2}^{2}+\bar{N} x_{1}+\bar{R}\right)$ is the model uncertainties. The output of neural network $\hat{f}(x)$ is used to approximate it. Therefore, considering (3), the control law can be chosen as

$$
Q=\bar{M}\left[-z_{1}-k_{2} z_{2}-k_{1}\left(x_{2}-\dot{r}(t)\right)+\ddot{r}(t)-\hat{f}(x)\right] .
$$

where $\hat{f}(x)$ can be expressed as follows:

$$
\hat{f}(x)=\hat{W}^{T} \phi .
$$

In which, $\hat{W}$ is the neural network weights matrix, $\phi=\left[\begin{array}{llll}\phi_{1} & \phi_{2} & \cdots & \phi_{6}\end{array}\right]^{T}$ are base functions of neural 
network. In addition, the uncertainties $f(x)$ can be expressed as

$$
f(x)=W^{* T} \phi+\varepsilon_{1} .
$$

where, $W^{*}$ is optimization weights matrix, $\varepsilon_{1}$ is the smallest approximation error of neural network.

Defining $\hat{\Lambda}=\operatorname{diag}\left[\begin{array}{ll}0 & \hat{W}\end{array}\right], \quad \Lambda=\operatorname{diag}\left[\begin{array}{ll}0 & W^{*}\end{array}\right]$ with $\|\Lambda\|_{F} \leq \Lambda_{M}$, in which, $\|\bullet\|_{F}$ denote the Frobenius norm. Let $\tilde{\Lambda}=\Lambda-\hat{\Lambda}$ and $z=\left[\begin{array}{ll}z_{1}^{T} & z_{2}^{T}\end{array}\right]^{T}$, then consider the following Lyapunov candidate function:

$$
V=\frac{1}{2} z^{T} z+\frac{1}{2} \operatorname{tr}\left(\tilde{\Lambda}^{T} \Sigma^{-1} \tilde{\Lambda}\right) \text {. }
$$

where $\Sigma$ is given by $\Sigma=\operatorname{diag}\left[\begin{array}{ll}0 & \Gamma\end{array}\right]$, in which $\Gamma$ is $6 \times 6$ diagonal positive definite matrix.

Choose the following adaptive law of neural network weights:

$$
\dot{\hat{\Lambda}}=\Sigma \Phi z^{T}-n \Sigma\|z\| \hat{\Lambda} .
$$

where, $n$ is positive real number and $\Phi=\left[\begin{array}{ll}0 & \phi^{T}\end{array}\right]^{T}$, $\| \bullet$ denote the 2-norm.

The derivative of (8) is

$$
\dot{V}=z^{T} \dot{z}+\operatorname{tr}\left(\tilde{\Lambda}^{T} \Sigma^{-1} \dot{\tilde{\Lambda}}\right) \text {. }
$$

Substituting (5) 、(6) and (7) into (10) yields $\dot{V}=-k_{1} z_{1}^{T} z_{1}-k_{2} z_{2}^{T} z_{2}+z_{2}^{T}\left[\left(W^{* T}-\hat{W}^{T}\right) \phi+\varepsilon_{1}\right]+\operatorname{tr}\left(\tilde{\Lambda}^{T} \Sigma^{-1} \dot{\tilde{\Lambda}}\right)$ Let $\quad \tilde{W}^{T}=W^{* T}-\hat{W}^{T} \quad$ then $\dot{V}=-k_{1} z_{1}^{T} z_{1}-k_{2} z_{2}^{T} z_{2}+z_{2}^{T}\left(\tilde{W}^{T} \phi+\varepsilon_{1}\right)+\operatorname{tr}\left(\tilde{\Lambda}^{T} \Sigma^{-1} \dot{\tilde{\Lambda}}\right) \quad$.

Defining $\quad K_{Z}=\operatorname{diag}\left[\begin{array}{ll}k_{1} I & k_{2} I\end{array}\right], \quad \varepsilon=\left[\begin{array}{ll}0 & \varepsilon_{1}^{T}\end{array}\right]^{T} \quad$ and assuming that $\|\varepsilon\|<\varepsilon_{N}$, there yields $\dot{V}=-z^{T} K_{Z} z+z^{T} \varepsilon+\operatorname{tr}\left(\tilde{\Lambda}^{T} \Sigma^{-1} \dot{\tilde{\Lambda}}+\tilde{\Lambda}^{T} \Phi z^{T}\right)$. As $\dot{\tilde{\Lambda}}=-\dot{\hat{\Lambda}}$, considering (9), the derivative of $V$ becomes

$$
\dot{V}=-z^{T} K_{Z} z+z^{T} \varepsilon+n\|z\| \operatorname{tr}\left(\tilde{\Lambda}^{T}(\Lambda-\tilde{\Lambda})\right) .
$$

According to Schwarz inequality $\operatorname{tr}\left(\tilde{\Lambda}^{T}(\Lambda-\tilde{\Lambda})\right) \leq\|\tilde{\Lambda}\|_{F}\|\Lambda\|_{F}-\|\tilde{\Lambda}\|_{F}^{2} \quad, \quad$ since $z^{T} K_{Z} z \geq k_{\min }\|z\|^{2}$, where $k_{\min }>0$ is the minimum eigenvalue of $K_{Z}$, then (11) becomes

$$
\dot{V} \leq-k_{\min }\|z\|^{2}+\varepsilon_{N}\|z\|+n\|z\|\left(\|\tilde{\Lambda}\|_{F}\|\Lambda\|_{F}-\|\tilde{\Lambda}\|_{F}^{2}\right) \leq-\|z\|\left[k_{\min }\|z\|-\varepsilon_{N}+n\left(\|\tilde{\Lambda}\|_{F}^{2}-\|\tilde{\Lambda}\|_{F} \Lambda_{M}\right)\right]_{\text {Figure 2. The tracking of }}^{\text {time(s) }} \theta_{2}
$$

In order to ensure $\dot{V}<0$, the inequality $k_{\min }\|z\|-\varepsilon_{N}+n\left(\|\tilde{\Lambda}\|_{F}^{2}-\|\tilde{\Lambda}\|_{F} \Lambda_{M}\right)>0 \quad$ should $\quad$ be guaranteed. Therefore, the inequality $\left(\varepsilon_{N}+\frac{n}{4} \Lambda_{M}^{2}\right) / k_{\min }<\|z\|$ can be derived. As a result, $\dot{V}<0$ can be ensured by choosing the suitable values of $n$ and $k_{\min }$. Therefore, the derived control law (5) can make $z_{1}$ and $z_{2}$ converge to zero exponentially asymptotically according to Lyapunov stability theorem. That is to say, the trajectory of work platform can follow the desired trajectory without vibration.

\section{IV、SIMULATION RESULTS}

The simulation results in Figure. 1 Figure. 6 are based on the following choice of design parameters and initial conditions:

$l_{1}=7.5 \mathrm{~m} \quad, \quad l_{2}=8.5 \mathrm{~m} \quad, m_{1}=650 \mathrm{~kg} \quad, m_{2}=550 \mathrm{~kg}$, $m=150 \mathrm{~kg}, d=0.05, c=0.03, m_{y 1}=20 \mathrm{~kg}, m_{y 2}=20 \mathrm{~kg}$,

$E I_{1}=6 \times 10^{8} \mathrm{~N} \cdot \mathrm{m}^{2}, \quad E I_{2}=5 \times 10^{8} \mathrm{~N} \cdot \mathrm{m}^{2}, \quad a_{10}=0.9 \mathrm{~m}$, $a_{11}=0.9 m \quad, \quad a_{12}=1.8 m, a_{21}=1.8 m, a_{22}=0.9 m$, the initial angular of two beams are $\theta_{1}=2.09 \mathrm{rad}$ and $\theta_{2}=0.52 \mathrm{rad}$, initial angular velocity $\dot{\theta}_{1}$ and $\dot{\theta}_{2}$ are zero.

Reference trajectory is designed as $r(t)=\left[\begin{array}{llllll}2 \pi / 3 & \pi t / 180 & 0 & 0 & 0 & 0\end{array}\right]^{T} \quad, \quad$ then $\dot{r}(t)=\left[\begin{array}{llllll}0 & \pi / 180 & 0 & 0 & 0 & 0\end{array}\right]^{T}$ $\ddot{r}(t)=\left[\begin{array}{llllll}0 & 0 & 0 & 0 & 0 & 0\end{array}\right]^{T}$.

In the control law (5), the design parameters are selected as $k_{1}=k_{2}=0.5$. In (6), the base functions of neural network are chosen as $\phi_{i}=\exp \left(-\frac{\|x\|^{2}}{2 \times 100^{2}}\right)$, $i=1,2, \cdots, 10$, in which, $x=\left[\begin{array}{ll}x_{1}^{T} & x_{2}^{T}\end{array}\right]^{T}$, the center of Gaussian function is set at 0 , the width is set at 100 . And the parameters in(9) can be set as: $n=0.01$, $\Gamma=100 \operatorname{diag}\left[\begin{array}{llllll}1 & 1 & 1 & 1 & 1 & 1\end{array}\right]$. In addition, initial weights are set to zero.

Fig .1 Fig .6 show the simulation results of applying the proposed neural network-based backstepping controller for the trajectory tracking control of work platform.
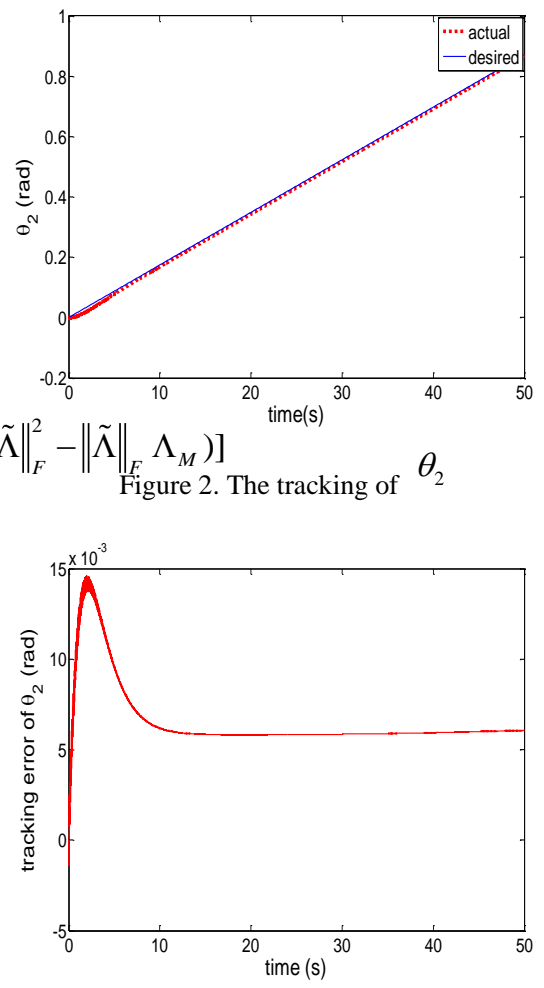

Figure 3. The tracking error of $\theta_{2}$ 


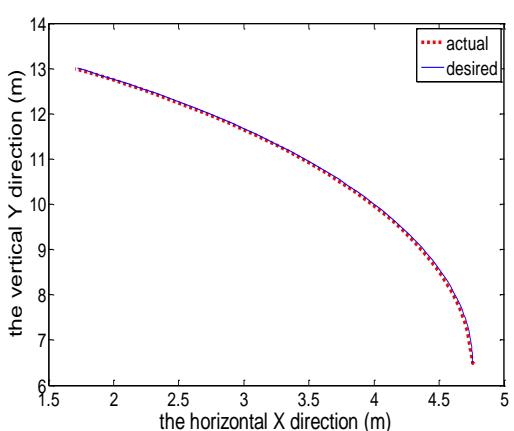

Figure 4 . The trajectory tracking of work platform

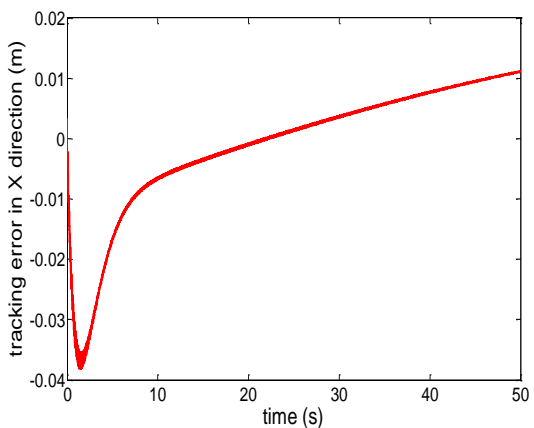

Figure 5. The trajectory tracking error

of work platform in the horizontal $\mathrm{x}$-direction

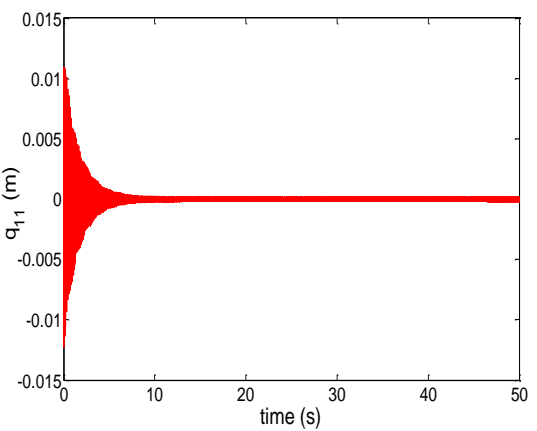

Figure 6. The deformation variable $q_{11}$ changing with time

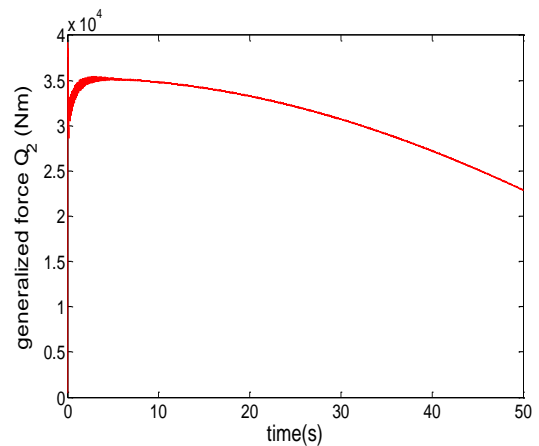

Figure 7. The regulation of generalized force $Q_{2}$

It can be seen from Fig .2 and Fig .4 that both the actual trajectory of $\theta_{2}$ and work platform can follow the desired trajectory closely by using the proposed neural network-based backstepping controller. Simultaneously, Fig .3 and Fig .5 demonstrate that the tracking errors are small, which reflect that the good tracking performance of the proposed controller. Fig. 6 is the deformation variable $q_{11}$ changing with time, It can be shown from Fig .6 that $q_{11}$ converges to small values with a transient, which means that the vibration is declined. As a result, the work platform can keep the steady movement along the desired trajectory. The regulation of generalized force $Q_{2}$, which is used for realizing the trajectory tracking control of work platform, is shown in Fig .7.

\section{V、CONCLUSION}

As neural network can approximate any nonlinear function with arbitrary accuracy, a neural network-based backstepping controller, which is the combination of backstepping control and neural network, is presented for trajectory tracking control of work platform when there exist model uncertainties. Furthermore, simulation results have shown that the proposed controller is an effective control scheme for suppressing vibration and attenuating tracking error of work platform in the case of model uncertainties. As a result, the trajectory of work platform can track the desired trajectory steadily.

\section{ACKNOWLEDGMENT}

This work was supported by Natural Science Foundation of Inner Mongolia (Grants No. 2012MS0907), and partly by

the National Natural Science Foundation of China

(Grant No.61370176 and No.61021004).

\section{REFERENCES}

[1] H. D. Hu, E. Li, X. G. Zhao, Z. Z. Liang and W. S. Yu, "Modeling and Simulation of Folding-Boom Aerial Platform Vehicle Based on the Flexible Multi-body Dynamics," 2010 IEEE International Conference on Intelligence Control and Information Processing (ICICIP 2010), 2010, pp.798-802.

[2] J. W. Huang and J. S. Lin, "Backstepping Control Design of a Single-Link Flexible Robotic Manipulator," Proceedings of the 17th IFAC World Congress, 2008, pp.11775-11780.

[3] F. S. Chen and J. S. Lin, "Nonlinear backstepping design of robot manipulators with velocity estimation feedback," Proceedings of the 5th Asian Control Conference, 2004, pp.351-356.

[4] H. D. Hu, E. Li, X. G. Zhao, M. Tan and W. S. Yu, "Backstepping Controller Design for the trajectory tracking control of work platform of Folding-Boom Aerial Platform Vehicle," 2010 IEEE International Conference on Robotics and Biomimetics (ROBIO 2010), 2010, pp.1641-1644.

[5] Y.-C. Chang and B.-S. Chen, "Robust tracking designs for both holonomic and nonholonomic constrained mechanical systems: adaptive fuzzy approach,” IEEE Trans. Fuzzy Systems, vol. 8(1) 2000, pp.46-65.

[6] B.-S. Chen, C.-H. Lee and Y.-C. Chang, "Hळ tracking design of uncertain SISO systems: adaptive fuzzy approach," IEEE Trans. Fuzzy Systems, vol. 4(1), 1996, pp.32-43.

[7] H. Lee and M. Tomizuka, "Robust adaptive control using a universal approximator for SISO nonlinear systems," IEEE Trans.Fuzzy Systems, vol. 8(1), 2000, pp.95-106.

[8] J.Park and I.W.Sandberg, "Universal approximation using radial-basis-function networks," Neural computation, vol. 3(1), 1991, pp.246-257.

[9] Shuzhi S.Ge and C Wang, "Direct adaptive NN control of a class of nonlinear systems," IEEE Transaction on neural networks, vol. 15(3), 2002, pp.214-221.

[10] Y P Zhang, P Y Peng and Z P Jiang, "Stable neural controller design for unknown nonlinear systems using backstepping," IEEE Transaction on neural networks, vol. 11(6), 2000 pp.1347-1360. 